\title{
Kim Williams und Michael J. Ostwald (Hrsg.): Architecture and Mathematics from Antiquity to the Future
}

\author{
Springer 2015, Volume I: From Antiquity to the 1500s, \\ XIX + 735 Seiten, ISBN 978-3-319-00136-4, €117,69; E-book \\ ISBN 978-3-319-00137-1, €93,08; Volume II: The 1500s to the \\ Future, XIX + 690 Seiten, ISBN 978-3-319-00142-5, €117,69, \\ E-book ISBN 978-3-319-00143-2, €93,08
}

\section{Michael Joachim}

Angenommen: 5. Januar 2021 / Online publiziert: 22. Januar 2021

(C) Der/die Autor(en) 2021

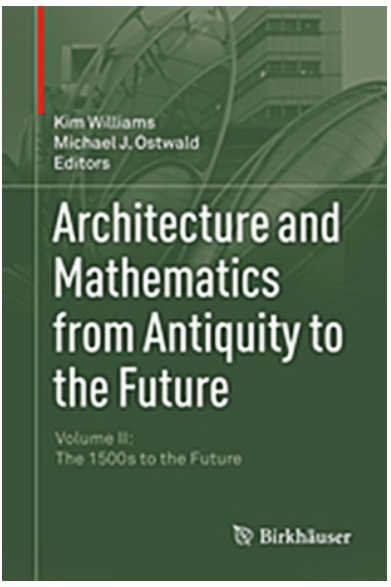

Das vorliegende zweibändige Werk gibt, wie es der Titel schon verspricht, einen guten Überblick über die zeitliche Entwicklung der vielfältigen Beziehungen zwischen den genannten Disziplinen: der Mathematik und der Architektur. Diese haben sich über die Jahrhunderte mehrfach deutlich gewandelt. Von der Antike bis in die Renaissance waren die Akteure in beiden Fachgebieten in der Regel Gelehrte, die sowohl im Bereich der Architektur als auch der Mathematik und oft auch in weiteren Disziplinen gut ausgebildet waren.

Ab dem 16. Jahrhundert führte dann das Aufkommen der Gilden und der technisch ausgerichteten Schulen dazu, dass man sich in der Befassung mit Architektur immer mehr auf rein architektur- bzw. bauspezifische Aspekte konzentrierte. Mit der Zeit entstand so eine gewisse Abgrenzung zur Mathematik. Aber auch Entwicklungen innerhalb der Mathematik trugen zu dieser Abgrenzung bei. Mit dem Aufkommen der reinen Mathematik und der rein theoretischen Betrachtung auch angewandter Fragestellungen konnte sich die Mathematik vergleichsweise schnell und unabhängig weiterentwickeln, während die Architektur wesentlich enger an die gesellschaftlichen Veränderungen und Strömungen im Bereich der Kunst gebunden war. Die zunehmende Spezialisierung in

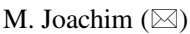

Universität Münster, Münster, Deutschland

E-Mail: joachim@wwu.de 
beiden Disziplinen machte es immer schwerer, in beiden Bereichen eine führende Rolle zu spielen.

Mit dem Aufkommen der Freiformarchitektur in der zweiten Hälfte des 20. Jahrhunderts und den in den letzten Jahren immer schnelleren Entwicklungen in der Digitalisierung haben sich Architektur und Mathematik wieder angenähert. Inzwischen spielen mathematische Konzepte nicht nur bei der Baufertigstellung, sondern auch beim Entwurf der Gebäude immer öfter eine entscheidende Rolle. Führende Architekturbüros stellen von daher heutzutage auch Mathematiker ein, und im Bereich der Modellierung und Optimierung befassen sich ganze Forschergruppen in mathematischen Instituten mit Fragestellungen, die aus der Architektur erwachsen.

Auch im Bereich des wissenschaftlichen Austausches gibt es heute einige Schnittstellen zwischen Architektur und Mathematik, doch es sind bislang nur wenige. Wer in seinem Browser nach wissenschaftlichen Artikeln sucht, die beide Bereiche betreffen, dem werden verschiedene angeboten. Doch meistens stammen die Artikel aus ein- und demselben Journal, dem Nexus Network Journal. Letzteres erscheint im Birkhäuser-Programm der Springer Nature Gruppe und ist mit inzwischen vier Ausgaben pro Jahr gut etabliert.

Doch das war nicht immer so. Der Gründung des Journals im Jahr 1999 ging zunächst eine Initiative voraus. Wissenschaftler, die im Grenzbereich zwischen Architektur und Mathematik arbeiteten, suchten den Austausch. In dieser Zeit war das Internet noch kaum entwickelt, und entsprechende wissenschaftliche Arbeiten konnten nur vereinzelt in wissenschaftlichen Journalen untergebracht werden, die aber mit ihren Profilen im Kern ganz andere Ziele verfolgten. Mit den Artikeln ,The cloisters of Hauterive" von Benno Artmann (The Mathematical Intelligencer 13(2) (1991), S. 44-49), „The Sacred Cut Revisited: The Pavement of the Baptistery of San Giovanni, Florence." von Kim Williams (The Mathematical Intelligencer 16(2) (1994), S. 18-24) und „Friedrich II and the love of geometry“ von Heinz Götze (The Mathematical Intelligencer 17(4)(1995), S. 48-57) boten die Autoren über die breite Leserschaft des Mathematical Intelligencers erstmals einen Kristallisationspunkt für die im Wesentlichen vereinzelt arbeitenden Wissenschaftler, und es entstand eine Interessengemeinschaft.

Zügig sollte mehr entstehen, und man organisierte deshalb die Konferenz „Nexus '96: Relationships between Architecture and Mathematics“, die 1996 in Fucecchio stattfand. Die Konferenz sollte den beabsichtigten breiteren Austausch bieten, und mit den Proceedings zur Konferenz erhielt man nun ein erstes gutes Medium, über das Forschungsergebnisse publiziert werden konnten. Die Nexus-Konferenzen fanden daraufhin alle zwei Jahre statt. Der Kreis der Interessenten wuchs schnell, und schon während der zweiten Konferenz wurde die Vision entwickelt, eine wissenschaftliche Zeitschrift für genau diesen Grenzbereich zu etablieren. Jedoch gab es nach der darauf folgenden Einführung des Nexus Network Journals im Jahre 1999 weiterhin noch die Proceedings zu den einzelnen Nexus-Konferenzen, und zwar noch bis 2008. Seit 2010 erscheinen die Proceedings in Sonderausgaben des Nexus Network Journals.

Doch die Artikel in den frühen Konferenz-Proceedings hatten Gewicht. Es waren die führenden Wissenschaftler der Szene, die hier über ihre Forschungsergebnisse berichteten. Die Artikel wurden vielfach zitiert, aber der Zugriff auf sie war 
oftmals nicht einfach. Die ersten Ausgaben der Konferenz-Proceedings waren teilweise schnell vergriffen, und so suchte man einen Weg, zumindest die oft zitierten Artikel der Allgemeinheit weiterhin zur Verfügung zu stellen. Statt sie nun einfach nachzudrucken, hatten die Herausgeber Kim Williams und Michael. J. Ostwald die Idee, die betreffenden Artikel chronologisch und thematisch zu sortieren und so mit dem Nachdruck gleichzeitig einen Überblick über die Entwicklung der Beziehungen zwischen Architektur und Mathematik von der Antike bis in die jüngste Zeit zu geben.

Dies ist nun genau der Inhalt der beiden vorliegenden Bände: Es sind gut sortierte, ausgewählte Nachdrucke aus den frühen Konferenz-Proceedings. Die Gesamtheit der ausgewählten Artikel ist so umfangreich, dass die Sammlung das Beabsichtigte auch leistet. Sie umfasst fast 100 Artikel, über 60 Bauwerke werden explizit beschrieben, es wird auf über 20 verschiedene theoretische Entwicklungen der Geometrie und des Designs eingegangen, und die Beispiele kommen aus vielen verschiedenen Kontinenten und umspannen zeitlich vier Jahrtausende.

Die Artikel, die die Zeit von den Anfängen in der Antike bis etwa 1500 n. Chr. betreffen, befinden sich im ersten der beiden Bände, die Zeit nach $1500 \mathrm{n}$. Chr. wird im zweiten Band behandelt. Für beide Bände haben die Herausgeber jeweils eine über zwanzigseitige Einleitung geschrieben, die zum einen die Entwicklung über die Zeit darstellt und zum anderen eine thematische Bündelung der Artikel vornimmt und reflektiert. Zusammen mit diesen Einleitungen entsteht in der Tat ein sehr rundes Bild der Geschichte der Architektur und der Mathematik, das die Artikel für sich alleine nicht hätten erreichen können.

Mit dem gewählten Konzept stellen die beiden Bände also - anders als es der Titel suggeriert - keine vollständige Enzyklopädie der geschichtlichen Entwicklung der Beziehungen zwischen beiden Fächern dar. Zwar erhält man, wie schon gesagt, durch die vorzüglichen Einleitungen der Herausgeber ein recht umfassendes Bild. Jedoch bieten die diversen Artikel darüber hinaus die Möglichkeit, einen viel tieferen und detaillierteren Einblick zu einzelnen Aspekten zu erhalten, als es in einem Werk möglich wäre, das dem Anspruch einer uniformen Betrachtung aller Zusammenhänge verpflichtet ist.

Für mich hat diese Mischung aus einer Gesamtschau und der Option, über einzelne Artikel bis an die Grenze der (aktuellen) Forschung zu kommen, die Lektüre der beiden Bände besonders lebendig gemacht. In einer auf vier Wochen in vier Semestern angelegten Fortbildung der Studienstiftung des deutschen Volkes (einem sogenannten wissenschaftlichen Kolleg) habe ich das zweibändige Werk daraufhin zum Thema gemacht. Wir verfolgten den mit den Einleitungen vorgegebenen Bogen über die Zeit und nutzten die einzelnen Artikel, um bei ausgewählten Themen in die Tiefe zu gehen. Zudem nahmen wir weitere Themen aus der Übersicht hinzu, die uns neugierig gemacht hatten. Die gleiche Lebendigkeit, die ich schon für mich aus der Lektüre ziehen konnte, war dann auch in der Arbeitsgemeinschaft zu spüren. Insofern möchte ich den Herausgebern ein ganz großes Lob zu dieser besonderen Ausgabe von Nachdrucken aussprechen.

Wie schon erwähnt, war die Motivation für die Herausgabe dieser beiden Bände vorrangig die Bereitstellung von viel zitierten Artikeln aus den Proceedings der ersten Nexus-Konferenzen. Da diese Proceedings das erste Printmedium waren, in 
dem regelmäßig Themen im Grenzgebiet zwischen Architektur und Mathematik besprochen wurden, veröffentlichten dort auch die für dieses Gebiet führenden Persönlichkeiten. Dazu gehören Leonard Eaton, Marco Frascari, Lionel March, Alberto Pérez-Gémez, Mario Salvadori, Robert Tavorner sowie der Herausgeber der Imagine Math-Reihe Michele Emmer, um nur einige zu nennen. Auch die Protagonisten der ersten Stunde, darunter die Herausgeberin Kim Williams sowie Benno Artmann von der TU Darmstadt und Heinz Götze vom Springer Verlag, lieferten Beiträge für die frühen Proceedings, die in die vorliegende Sammlung mit aufgenommen wurden. Natürlich wird nicht jeder Leser, jede Leserin alle Artikel des zweibändigen Werks gleichermaßen interessant finden, aber es ist für jeden und jede mit einer Neugierde für das Themenfeld sicher etwas dabei.

Exemplarisch möchte ich ein paar Thematiken nennen, die in den Beiträgen aufgenommen wurden. Mich persönlich hat zum Beispiel der Artikel von Heinz Götze sehr angesprochen. Dort stellt der Autor mathematische Überlegungen zum Castel del Monte in Apulien an, das seit 1996 Teil des Weltkulturerbes ist. Letzteres wurde im Auftrag des deutschen Staufferkaisers Friedrich II. im 13. Jahrhundert erbaut. Friedrich II. interessierte sich nachweislich sehr für Mathematik, und das Bauwerk weist offenkundig viele mathematisch initiierte Details auf. Es gibt zudem Vermutungen, die besagen, dass das ganze Gebäude ein reiner Kunstbau sei.

Ein weiterer Artikel der Sammlung, den wir im Übrigen auch bei dem oben erwähnten wissenschaftlichen Kolleg in den Fokus genommen haben, ist eine Arbeit über Muqarnas von Yvonne Dold-Samplonius und Silvia Hamsen. Murqarnas sind aufwändige Zierelemente der persischen Architektur, mit denen ein oberer Abschluss einer Nische oder ein Gewölbe ausgeschmückt werden. Die Muqarnas entstanden in der Mitte des 10. Jahrhunderts als Architekturelement und verbreiteten sich rasch über das gesamte damalige islamische Herrschaftsgebiet. In dem Artikel wird die zugrundeliegende mathematische Struktur der Muqarnas analysiert, und es wird aufgezeigt, wie man sie mit dem Einsatz von Computern rekonstruieren kann.

Leonard Eaton analysiert in einem anderen Artikel die Geometrie der Fenster im Meyer May House von Frank Lloyd Wright, und in einem weiteren Artikel der Reprint-Sammlung erklärt der aktuelle Ars legendi-Preisträger Ulrich Kortenkamp die mathematischen Hintergründe zu einem Architektenentwurf der Firma Mettler zur Pflasterung des Berliner Alexanderplatzes mittels einer Penrose-Parkettierung, an dessen Umsetzung er beteiligt war. Die Beispiele sollen verdeutlichen, welche Bandbreite und welche Vielfalt das zweibändige Werk aufweist.

Natürlich decken die beiden Bände nicht alle Themenfelder des Grenzgebiets vollständig ab. Beispielsweise fehlen Artikel zu Brunelleschi, der zum einen als Bauherr der Kuppel von Santa Maria del Fiore in Florenz, aber vor allem für die konsequente Einführung der Perspektive in der Architektur verantwortlich war. Auch werden die auffälligen Bezüge der Bauwerke von Otto Frei (dem Erbauer des Münchener Olympiastadions) zur Theorie der Minimalflächen nur beiläufig in einem Aufsatz von Michele Emmer erwähnt, und die Freiformarchitektur als Ganzes mit ihren bekannten Vertretern wie Norman Foster, Frank Gehry oder Zaha Hadid bleiben gänzlich ungenannt.

Andererseits werden andere Aspekte überproportional in Szene gesetzt. So gibt es beispielsweise alleine drei Artikel zur Architekturtheorie des Leon Battista Alberti. 
Und auch Werke von Frank Lloyd werden gleich in drei verschiedenen Werken in den Fokus genommen. Aber dies liegt natürlich auch darin begründet, dass es sich hier um eine Auswahl von Nachdrucken handelt und nicht jedes Thema im Grenzbereich der Architektur und der Mathematik schon Thema eines Nexus-Konferenzvortrages gewesen sein kann. Naturgemäß findet man in den beiden Bänden von daher auch keine Artikel zu den ganz jungen und spannenden Entwicklungen, wie zum Beispiel zum Entwerfen von Gebäuden mittels moderner digitaler Entwicklungstools wie der Grashopper-Anwendung. Jedoch ist es den beiden Herausgebern gelungen, mit der Aufbereitung der Artikel ein wunderschönes Sammelsurium von Informationen zusammenzustellen, das auch einem Laien das wissenschaftliche Grenzgebiet zwischen Architektur und Mathematik eröffnen kann. Viele Artikel erfahren durch das hochwertige Format eine besondere Wertschätzung und erreichen so hoffentlich einen erweiterten Leserkreis. Die Qualität der Artikel verdient dies allemal.

Funding Open Access funding enabled and organized by Projekt DEAL.

Open Access Dieser Artikel wird unter der Creative Commons Namensnennung 4.0 International Lizenz veröffentlicht, welche die Nutzung, Vervielfältigung, Bearbeitung, Verbreitung und Wiedergabe in jeglichem Medium und Format erlaubt, sofern Sie den/die ursprünglichen Autor(en) und die Quelle ordnungsgemäß nennen, einen Link zur Creative Commons Lizenz beifügen und angeben, ob Änderungen vorgenommen wurden.

Die in diesem Artikel enthaltenen Bilder und sonstiges Drittmaterial unterliegen ebenfalls der genannten Creative Commons Lizenz, sofern sich aus der Abbildungslegende nichts anderes ergibt. Sofern das betreffende Material nicht unter der genannten Creative Commons Lizenz steht und die betreffende Handlung nicht nach gesetzlichen Vorschriften erlaubt ist, ist für die oben aufgeführten Weiterverwendungen des Materials die Einwilligung des jeweiligen Rechteinhabers einzuholen.

Weitere Details zur Lizenz entnehmen Sie bitte der Lizenzinformation auf http://creativecommons.org/ licenses/by/4.0/deed.de. 